\title{
Efeitos de diferentes modelos de periodização do treinamento aeróbio sobre parâmetros cardiovasculares, metabólicos e composição corporal de bombeiros militares
}

CDD. 20.ed. 616.12

796.073

Anselmo José PEREZ*

\section{Resumo}

0 objetivo do estudo foi analisar o efeito de programa de treinamento aeróbio sobre parâmetros cardiovasculares, metabólicos e de composição corporal comparando diferentes periodizações. Sessenta e cinco bombeiros militares/ES ( $25,9 \pm 0,6$ anos) foram divididos em quatro grupos: controle (Gcon, $n=$ 15) sem tratamento; ondulatório (Gond, $n=18$ ), escalonado (Ges, $n=17$ ), e crescente (Gcres, $n=15$ ), e treinaram 13 semanas, três sessões semanais de 30 minutos e intensidades de 60\% a 90\% FCmáx. A frequência cardiaca (FC) foi controlada em todas as sessões. Antes e após o programa foram realizadas avaliações antropométricas e teste cardiopulmonar (Aerosport TEEM-100). Houve redução no percentual de gordura, bradicardia em repouso e da FC submáxima com aumento do consumo de oxigênio, e redução do duplo produto, da PAD em repouso para os três grupos treinados, sem vantagens adicionais para o Gcres. Esses resultados sugerem que programas de treinamento aeróbio com distribuição de intensidade ao longo das semanas de forma escalonada e/ou ondulatória podem ser mais apropriados para indivíduos não atletas.

PalavRas-chave: Treinamento físico; Periodização; Frequência cardiaca; Saúde cardiovascular; Bradicardia; Adaptação fisiológica.

\section{Introdução}

Nos últimos anos diversos programas de treinamento físico têm sido propostos, principalmente para a maioria da população considerada não atletas e que procuram praticar atividades físicas de forma regular, agradável e sem fins competitivos. Segundo HASKELL et al. ${ }^{1}$ a atividade física regular bem planejada está associada a uma melhor saúde e qualidade de vida. Além disso, o treinamento físico tem sido proposto como método terapêutico não farmacológico adequado para manutenção e prevenção de doenças cardiovasculares ${ }^{2-6}$.

$\mathrm{O}$ treinamento aeróbio não deve ser visto apenas para a preparação de atletas de alto rendimento (níveis profissionais e semiprofissionais), e embora os seus desempenhos e padrões físicos tenham se tornado exemplos de saúde para os atletas amadores e para os não atletas, seus programas de treinamento não precisam ser completamente copiados, até mesmo porque muita coisa é intuitiva ${ }^{7}$. Atletas têm o foco em competições e desempenhos máximos, podendo exceder os estímulos do treinamento, tendo como consequência lesōes musculoesqueléticas prejudiciais à saúde.

Há necessidade então de cuidados para o uso de metodologias aplicadas àquelas pessoas que querem melhorar o seu condicionamento físico para o seu trabalho e saúde, e as recomendaçôes incluem a dinâmica de treinamento que envolve a manipulação da carga por meio das variáveis: intensidade, duração e frequência de estímulos, além do tipo do exercício ${ }^{1,8-9}$. Porém, apesar das discussões da relação exercício e saúde e da necessidade de uma dose-resposta apropriada, nada em relação a periodização de cargas aeróbias tem sido sugerido para pessoas saudáveis, não atletas.

Observaçōes empíricas sobre o planejamento e controle das sessões de treino de atletas possibilitaram identificar que para o aumento de cargas durante as semanas (periodização) os métodos por degrau, ou seja, com elevação em forma de escada, e os do tipo ondulatório, ou seja, em forma de ondas crescentes, são mais eficientes do que os métodos linear 
(aumento contínuo até o máximo e manutenção) e contínuo (sempre a mesma carga) ${ }^{10-13}$.

Periodização de treinamento significa a subdivisão do programa sazonal em períodos menores e ciclos de treinamento $^{13}$. A base da teoria tradicional de periodização de treinamento inclui, entre outras coisas, uma elucidação geral de carga e de recuperação tendo em vista o conceito de supercompensação. Assim, uma progressão gradual, como sugerido pelo Colégio Americano de Medicina Esportiva- $\mathrm{ACSM}^{5}$, não garante que ocorram fases de estímulo e recuperação nos microciclos (semanas), pois ela pode ser feita de forma linear, somente com aumento da intensidade, ou permanecendo com a mesma intensidade e/ou mesmo volume.

Esta preocupação é porque, frequentemente, os programas de atividade física regular são iniciados sem uma dosagem adequada dos estímulos aeróbios, não respeitando nem o princípio da sobrecarga (progressão) e nem as próprias características individuais. Isto pode rapidamente levar a um estado de "overtraining", ou seja, um estado de fadiga ${ }^{14}$, e afastar o praticante do programa de treinamento precocemente.

\section{Método}

\section{Amostra}

Noventa e dois voluntários do sexo masculino foram avaliados inicialmente. Todos pertenciam ao Corpo de Bombeiros Militar/ES, sendo 54\% alunos do Curso de Formação de Soldados, e os demais soldados e oficiais. Destes, $65 \%$ realizou o segundo teste após o encerramento de um programa de treinamento aeróbio (PTA). Foram incluídos somente aqueles que realizaram no mínimo $85 \%$ das sessões e que preencheram rigorosamente os itens referentes ao controle das sessōes. No grupo controle não foram incluídos homens que se engajaram em alguma atividade física regular ou mudaram seus hábitos de vida, como mudança de função no quartel, por exemplo. Além disso, o atual programa de treinamento não estava somado às atividades militares de rotina de treinamento. Esse grupo de homens permaneceu realizando somente o PTA e a rotina diária fazia parte das atividades programadas no quartel, incluindo: instrução, alimentação, horários de trabalho, estudo e lazer, além do treinamento aeróbio.

Eles responderam um questionário inicial para estratificação dos fatores de risco e passaram por medidas antropométricas de peso e altura (balança
Apenas um trabalho foi encontrado, até o momento, em que se considerou a influência da distribuição das cargas semanais de treinamento no rendimento aeróbio de não atletas ${ }^{15}$.

A hipótese deste trabalho é que programas regulares de atividades físicas aeróbias possam mostrar vantagens em seus resultados com uma organização de cargas periodizadas de forma ondulatória ou crescente, ao invés de utilizar método linear ou contínuo, potencializando os benefícios relacionados à saúde.

Assim, o objetivo foi estudar as respostas fisiológicas em homens saudáveis, submetidos a um programa de treinamento predominantemente aeróbio, pela caminhada/corrida, com diferentes estruturaçōes (distribuições e aumentos) de intensidades na periodização, analisando e comparando os efeitos das periodizações em relação aos parâmetros relacionadas à saúde: composição corporal, frequência cardíaca (FC), pressão arterial sistólica (PAS) e pressão arterial diastólica (PAD), e duplo produto (DP), em repouso, esforço e recuperação, e consumo de oxigênio $\left(\mathrm{VO}_{2}\right)$ em nível submáximo e máximo.

com toesa, da marca Welmy (São Paulo/SP, Brasil), $-0,1 \mathrm{~kg}$ e $0,1 \mathrm{~cm}$, respectivamente); medida de dobras cutâneas: tríceps (TR), subescapular (SB), supra-ilíaca $(\mathrm{SI})$, abdominal $(\mathrm{AB})$, coxa $(\mathrm{CX})$ e perna (PM), com compasso da marca Cescorf $(0,1$ $\mathrm{mm}$ ) (Porto Alegre/RS, Brasil), onde se calculou o índice de massa corporal (IMC - kg/altura ${ }^{2}$ ), e o percentual de gordura $(\% \mathrm{G})$, além do somatório das seis dobras ( $\Sigma 6 \mathrm{DC})$.

Tratava-se de homens normotensos (PAS $119 \pm$ 13 e PAD $75 \pm 8 \mathrm{mmHg}$ ), com idade entre $20 \mathrm{e}$ 47 anos (25,9 \pm 0,64 anos), sendo aleatoriamente divididos em quatro grupos denominados: grupo controle (Gcon, $n=25)$, o qual não recebeu nenhum tratamento em relação à variável independente (treinamento aeróbio) ou qualquer tipo de atividade física regular; grupo crescente (Gcres, $n=22$ ), que recebeu cargas lineares, ou seja, sempre com aumento de carga da menor intensidade até a maior; e dois grupos, ondulatório (Gond, $\mathrm{n}=23$ ) e escalonado (Ges, $n=22$, que receberam cargas não lineares, ou seja, com estabilizaçōes ou reduçôes de cargas durante as semanas (microciclos), na progressão da menor para a maior intensidade. O PTA foi composto de 13 semanas como apresentado no QUADRO 1. 
Todos conheciam os objetivos do trabalho e concordaram com a participação no mesmo, assinando um termo de consentimento, o qual foi aprovado pelo Comitê de Ética, do Centro de Ciências da Saúde - CCS/UFES (Parecer $n^{\circ}$ 1102/98).

QUADRO 1 - Distribuição das cargas de treinamento aeróbio (caminhada/corrida) em relação ao percentual da intensidade da FCmáx. para cada grupo durante as 13 semanas.

\begin{tabular}{|c|c|c|c|c|c|c|c|c|c|c|c|c|c|}
\hline Meso & \multicolumn{4}{|c|}{ Introdutório } & \multicolumn{4}{|c|}{ Desenvolvimento 1} & \multicolumn{5}{|c|}{ Desenvolvimento 2} \\
\hline Micro & 1 & 2 & 3 & 4 & 5 & 6 & 7 & 8 & 9 & 10 & 11 & 12 & 13 \\
\hline Gcres & $\begin{array}{l}60 / \\
65 \%\end{array}$ & $\begin{array}{l}65 / \\
70 \%\end{array}$ & $\begin{array}{l}70 / \\
75 \%\end{array}$ & $\begin{array}{l}75 / \\
80 \%\end{array}$ & \multicolumn{6}{|c|}{$80 / 85 \%$} & \multicolumn{3}{|c|}{$85 / 90 \%$} \\
\hline Gond & $\begin{array}{l}60 / \\
65 \%\end{array}$ & $\begin{array}{l}65 / \\
70 \%\end{array}$ & $\begin{array}{l}70 / \\
75 \%\end{array}$ & $\begin{array}{l}60 / \\
65 \%\end{array}$ & $\begin{array}{l}65 / \\
70 \%\end{array}$ & $\begin{array}{l}70 / \\
75 \%\end{array}$ & $\begin{array}{l}75 / \\
80 \%\end{array}$ & $\begin{array}{l}60 / \\
65 \%\end{array}$ & $\begin{array}{l}75 / \\
80 \%\end{array}$ & $\begin{array}{l}80 / \\
85 \%\end{array}$ & \multicolumn{3}{|c|}{$85 / 90 \%$} \\
\hline Ges & \multicolumn{2}{|c|}{$60 / 65 \%$} & \multicolumn{2}{|c|}{$65 / 70 \%$} & \multicolumn{3}{|c|}{$65 / 70 \%$} & \multicolumn{3}{|c|}{$75 / 85 \%$} & \multicolumn{3}{|c|}{$85 / 90 \%$} \\
\hline
\end{tabular}

Gcres = grupo que recebeu cargas crescentes; Gond = grupo que recebeu cargas ondulatórias;

Ges = grupo que recebeu cargas escalonadas.

Obs: Houve variação de $5 \%$ na intensidade em cada micro e em cada sessão de treinamento.

\section{Plano de treinamento aeróbio (PTA)}

Dependendo da intensidade planejada para a sessão e das condições individuais, o PTA era realizado por caminhada em seu início e corrida na sequência, com três sessões semanais de 50 minutos (10 minutos de aquecimento, 30 minutos de estímulo aeróbio e 10 minutos de alongamento final), e duração total de 13 semanas. $O$ método de treinamento utilizado nas sessões foi o contínuo.

O mesociclo introdutório objetivou adaptação do sistema locomotor passivo, principalmente nas duas primeiras semanas. Os outros dois mesociclos, de desenvolvimento, visaram às adaptaçóes fisiológicas.

A FC foi utilizada para o planejamento e controle da intensidade de esforço (Polar ${ }^{\circledR}$ Filland - beat, accurex $)^{16}$, como habitualmente padronizado ${ }^{1}$. Os três grupos treinados iniciaram o PTA com intensidade de 60-65\% da FCmáx. atingida no teste cardiopulmonar e terminaram o programa com estímulos entre 85 e $90 \%$ da FCmáx., porém, com a periodização das intensidades distribuídas de forma diferente como visto no QUADRO 1. Dentro da faixa de percentual da FCmáx. para treinamento individual, foi feita uma relação com a frequência cardíaca de reserva e mantida uma proporcionalidade em cada sessão até os $90 \%$ da FCmáx., o que respeitava estímulos entre $80-85 \%$ da reserva ${ }^{17}$.

Assim, Gond aumentou de 60\% até $85-90 \%$ da FCmáx., de forma ondulatória; Gcres, também de 60 até $85-90 \%$ da FCmáx., mas sem períodos de recuperação nas semanas e de forma linear, e Ges com semanas de manutenção de carga. Enquanto Gond e Ges receberam intensidades de 85\% apenas nas três últimas semanas, Gcres realizou as sessões nessa intensidade desde a quinta semana de treino, ou seja, seis semanas a mais do que os outros dois grupos e essa diferença proposital objetivou a confirmação da hipótese substantiva.

\section{Coleta de dados \\ e protocolo do teste cardiopulmonar}

Antes da realização dos testes cardiopulmonares, os indivíduos eram avisados sobre a não ingestão de grandes refeições, abstinência do uso de bebidas alcoólicas e de tabaco, pelo menos três horas antes do teste de esforço, além de evitar esforço físico demasiado tanto no dia anterior como no dia do exame.

Todos realizaram um eletrocardiograma (ECG) de repouso, nas 12 derivaçóes convencionais e permaneciam sentados durante aproximadamente cinco minutos, sendo orientados sobre os procedimentos de realização e interrupção do teste.

Em seguida, o indivíduo era acoplado ao pneumotacômetro e ficava em pé durante três minutos (préesforço e adaptação). O fim do teste ocorreu no exato momento em que o avaliado pedia para interromper o teste. Foi realizado um período de recuperação e eles permaneciam caminhando por três minutos, numa inclinação de $0 \%$ e velocidade de $2,4 \mathrm{~km} / \mathrm{h}$.

Os testes foram realizados em uma esteira rolante (Inbramed - modelo KT 10200, Porto Alegre/RS, Brasil), com monitorização contínua da FC através do intervalo R-R obtido pelo ECG, utilizando derivações simultâneas em MC5, D2M e V2M (sistema TEB, modelo SM 400, - Inbramed, Porto Alegre/RS, Brasil), e temperatura controlada entre 20 e 25 graus. A ventilação minuto (VE) e a fração expirada de oxigênio e dióxido de carbono foram continuamente medidas através de calorimetria indireta de circuito aberto (TEEM $100^{\circledR}$ Total Metabolic Analysis System, Aerosport ${ }^{\circledR}$, Ann Arbor, MI., EUA), sistema metabólico comercial previamente validado ${ }^{18-19}$. Os avaliados utilizaram um clipe 
de nariz e um pneumotacômetro de fluxo médio (Hans Rudolph Inc ${ }^{\circledR}$, Kansas City, MO, EUA). O consumo de oxigênio por minuto $\left(\mathrm{VO}_{2}\right)$ e a eliminação de gás carbônico por minuto $\left(\mathrm{VCO}_{2}\right)$ foram apresentados a cada 20 segundos. A unidade do Aerosport TEEM 100 foi calibrada pelo método GASCAL + ZERO (calibração de circuito-fechado), através de gás de calibração (cilindro de $\% \mathrm{O}_{2}$ e $\% \mathrm{CO}_{2}$ original, fornecido pelo fabricante), permitindo uma nova calibragem antes de cada teste. As informações do medidor de voltagem relativas a fluxo, $\mathrm{O}_{2}, \mathrm{CO}_{2}$ e temperatura eram conferidas e, caso necessário, realizado outro processo de calibração. Durante a colocação do pneumotacômetro e durante todo o teste (protocolo de Bruce), um cuidado especial foi tomado para evitar vazamentos.

Os critérios para aceitar o teste como máximo seguiram orientação do proposto na literatura ${ }^{20}$ que se resumiam em: a) exaustão voluntária; b) FCmáx. atingida no teste estar pelo menos a 90\% da prevista para idade (220-idade); c) uma razão de troca respiratória (RTR) igual ou acima de 1,$1 ; \mathrm{d}$ ) consumo máximo de oxigênio ${ }^{21}$.

\section{Análise dos resultados}

Os resultados absolutos antes e ao final do PTA, incluindo as diferenças entre as médias (deltas - $\Delta$

\section{Resultados}

\section{Características etárias e de composição corporal}

Os resultados de média de idade, peso, altura, $\Sigma 6 \mathrm{DC}$ e $\% \mathrm{G}$ encontram-se na TABELA 1 . Apesar de Gcon ter idade significativamente maior do que os outros grupos, as variáveis antropométricas eram semelhantes antes do início do PTA. Os três grupos treinados reduziram significativamente o somatório de dobras cutâneas e o percentual de gordura. em cada estágio do teste cardiopulmonar), foram comparados por análise de variância (ANOVA) para medidas completamente randomizadas, de uma via ou duas vias, de acordo com as especificidades de cada variável, e foi usado o teste Tukey para encontrar as diferenças entre os grupos. Assim, nas comparaçôes de todas as variáveis dependentes: composição corporal, PAS e PAD, DP, e tempo total ( $\mathrm{T}$ - tempo total do teste sustentado pelo indivíduo), utilizou-se ANOVA de uma via. As variáveis dependentes medidas durante a fase de repouso e todas as fases do teste, ou seja, $\mathrm{VO}_{2}, \mathrm{FC}$ e DP, foram comparados os quatro grupos aplicando ANOVA de duas vias, considerando o Fator A os estágios, e Fator B os grupos Gcon, Gond, Gcres e Ge.

Dentro de cada grupo, as comparaçóes das variáveis dependentes (FC, PAS, PAD de repouso e $\mathrm{DP}$ de repouso, $\mathrm{VO}_{2}$ pico, e $\mathrm{T}$ ) nas fases do teste foram feitas utilizando-se teste $\mathrm{t}$ de Student para amostras pareadas.

Em todas as análises estatísticas o nível de rejeição da hipótese nula escolhido foi de $\mathrm{p}<0,05$. Nos casos em que os valores de probabilidade encontrados foram menores, escolheu-se apresentar aqueles possíveis de arredondamento em $\mathrm{p}<0,01$. Os cálculos estatísticos foram realizados pelo programa GB-Stat, versão 6.5 for Windows.

\section{Sessões de treinamento}

As FC durante as 33 sessões variaram dentro do planejado para todos os grupos e as distâncias percorridas foram de $2700 \mathrm{~m}$ até $8500 \mathrm{~m}$, do início até o fim do programa de treinamento, com velocidades entre $111 \pm$ 3 a $183 \pm 4 \mathrm{~m} \cdot \mathrm{min}^{-1}$ (FIGURA 1). Houve uma queda na velocidade média desenvolvida nas sessões para os três grupos treinados na penúltima semana, apesar do aumento da FC, sendo mais acentuada para o Gcres. 
TABELA 1 - Características etárias e de composição corporal, antes e após um programa de treinamento aeróbio, de 13 semanas, de três grupos de bombeiros militares/ES, que receberam diferentes periodizações de cargas, e grupo controle.

\begin{tabular}{lcccccccc}
\hline Grupos & \multicolumn{2}{c}{ Gcon $(15)$} & \multicolumn{2}{c}{ Gond (18) } & \multicolumn{2}{c}{ Gcres (17) } & \multicolumn{2}{c}{ Ges (15) } \\
Variável & antes & após & antes & após & antes & após & antes & após \\
\hline Idade (anos) & $30 \#$ & $30 \#$ & 25 & 25 & 25 & 25 & 22 & 22 \\
Peso $(\mathrm{kg})$ & 72,0 & 72,2 & 70,3 & 69,7 & 69,7 & 69,2 & 68,7 & 68,5 \\
Altura $(\mathrm{m})$ & 1,77 & 1,77 & 1,80 & 1,80 & 1,76 & 1,76 & 1,77 & 1,75 \\
IMC $\left(\mathrm{kg} / \mathrm{m}^{2}\right)$ & 23 & 23 & 22 & 22 & 23 & 22 & 22 & 22 \\
I6DC $(\mathrm{mm})$ & 83 & 85 & 68 & $58^{*}$ & 65 & $59^{*}$ & 74 & $60^{*}$ \\
$\% \mathrm{G}$ & 16,3 & 16,4 & 11,0 & $9,8^{*}$ & 12,1 & $10,8^{*}$ & 12,6 & $10,5^{*}$ \\
\hline
\end{tabular}

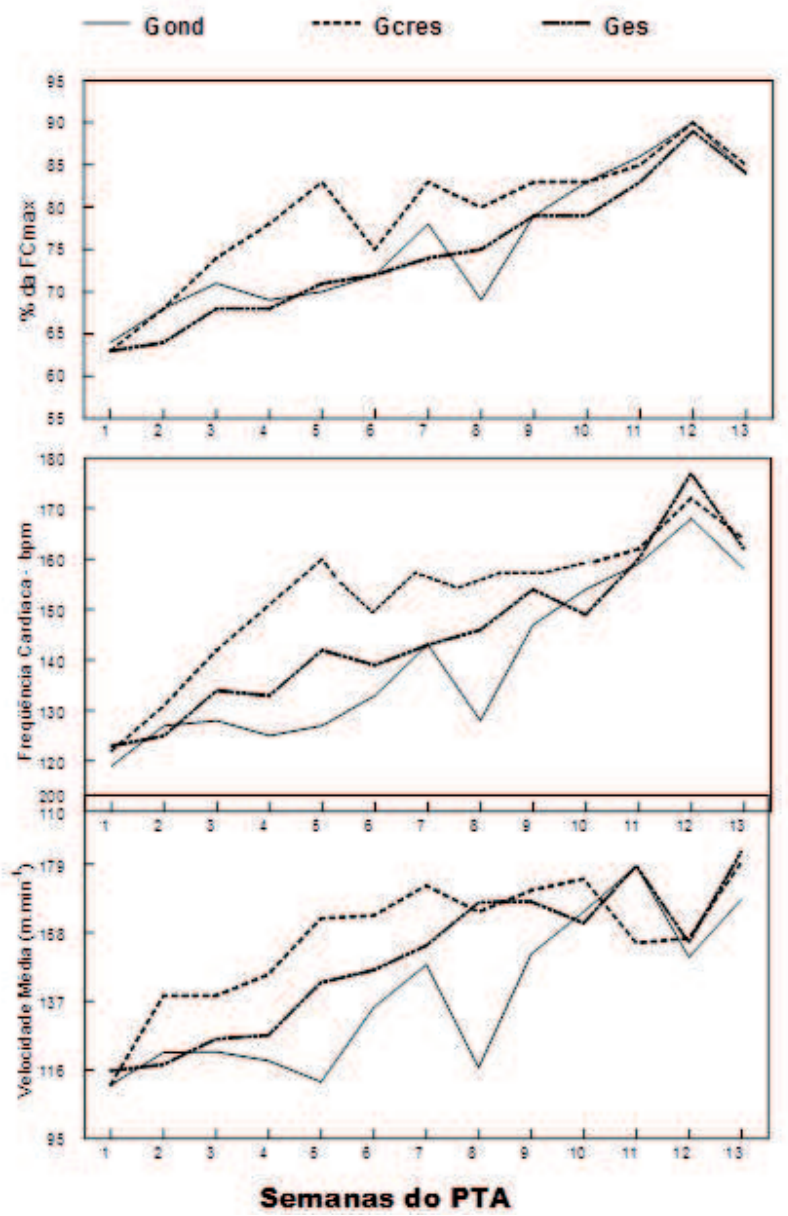

No painel superior porcentagem da FCmáx.; no painel central a média de FC atingida de acordo com $0 \%$ da FC planejada (controlada pelo MFC), e ;

no painel inferior a média da velocidade.

FIGURA 1 - Desempenho dos grupos em programa de treinamento aeróbio de 13 semanas (PTA) de acordo com a distribuição de cargas. 


\section{Comparações entre os grupos antes - início do PTA}

Antes de iniciar o PTA os grupos eram semelhantes em relação ao $\mathrm{VO}_{2}$, FC, T, DP, PAS e PAD. Exceçôes de diferenças significativas foram observadas no $\mathrm{VO}_{2}$ pico entre Ges vs Gcon e na PAS (Gond < Gcres e Gcon; Ges < Gcres), TABELAS 2 e 3.

\section{Comparações entre os grupos após - final do PTA}

Para o Gcon foram encontradas diferenças significativamente menores nas variáveis $\mathrm{Te}_{\mathrm{VO}}$ pico em relação ao Gond e Ges (TABELA 2), e para FC em repouso comparado com Gcres e Ges.

A PASfim do Ges foi significativamente maior quando comparada ao Gond e a Gcon, o mesmo acontecendo para Gcres em relação a Gond e Gcon. Também, foram encontradas diferenças na PAD entre Gcon vs Gcres e Ges, sendo o primeiro significativamente maior do que os outros (TABELA 3).

O consumo indireto de oxigênio pelo miocárdio, representado pelo DP, foi significativamente maior para os três grupos treinados quando comparados ao Gcon.

Quando comparados os quatro grupos, houve similaridade entre os resultados durante a recuperação para todas as variáveis estudadas.

\section{Efeito do treinamento: comparação do teste antes vs após o PTA dentro de cada grupo e intergrupos}

Nas TABELAS 2 e 3 observa-se que o Gcon não apresentou diferenças significativas entre nenhuma das variáveis estudadas no teste antes e após o PTA, nem em repouso e pré-esforço, enem nas fases de esforço e recuperação. $\mathrm{O} \mathrm{VO}_{2}$ pico aumentou significativamente para os três grupos treinados, sendo os maiores percentuais para o Ges. Nos esforços submáximos, um $\mathrm{VO}_{2}$ significativamente maior ( $8 \%$ ) foi encontrado no quarto estágio para o Ges, havendo aumento não significativo nesse estágio para os outros dois grupos treinados. Apenas nos estágios 1 e 2 valores menores de $\mathrm{VO}_{2}$ foram observados para o teste após o PTA, mas sem diferenças significativas. Isso ocorreu para Gond e Ges no estágio 1 e para Gcres e Ges no estágio 2, com maiores percentuais para o Ges.

Os valores durante a fase de recuperação dos testes cardiopulmonares antes e após não foram diferentes estatisticamente.

$\mathrm{O} \mathrm{T}$ foi aumentado nos três grupos treinados, porém sem diferenças estatisticamente significativas, com aumentos percentuais de 3\% a 7\%.

Nos estágios 3, 4 e no pico, encontraram-se $\Delta$ com diferenças significativas entre o Gcon comparado aos grupos Gond, Gcres e Ges, para o $\mathrm{VO}_{2}$. Os aumentos não foram diferentes entre os grupos treinados, apesar de uma tendência maior para o Ges (FIGURA 2).
Valores apresentados como média \pm EPM;

$\mathrm{VO}_{2}=$ Consumo de oxigênio;

$\mathrm{T}=$ tempo total suportado quando somado o tempo de todos os estágios.

$\# p<0,01$;

${ }^{*} p<0,05$ - Comparação antes vs após programa de treinamento - teste $t$ pareado.

TABELA 2 - Resultados de consumo de oxigênio e o tempo total medidos em teste cardiopulmonar, antes e após PTA de 13 semanas em três grupos de bombeiros que receberam diferentes periodizações de intensidades de cargas.



368 • Rev Bras Educ Fís Esporte, (São Paulo) 2013 Jul-Set; 27(3):363-76 
TABELA 3 - Resultados de FC, PA e DP medidos em teste cardiopulmonar, antes e após PTA de 13 semanas em três grupos de bombeiros militares/ES, que receberam diferentes periodizações de intensidades de cargas.

\begin{tabular}{|c|c|c|c|c|c|c|c|c|c|c|c|c|c|}
\hline \multirow{2}{*}{ 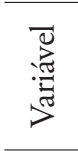 } & \multirow{2}{*}{$\begin{array}{l}\text { Grupos } \\
\text { Estágio }\end{array}$} & \multicolumn{3}{|c|}{$\operatorname{Gcon}(\mathrm{n}=15)$} & \multicolumn{3}{|c|}{ Gond $(\mathrm{n}=18)$} & \multicolumn{3}{|c|}{ Gcres $(\mathrm{n}=17)$} & \multicolumn{3}{|c|}{ Ges $(n=15)$} \\
\hline & & Antes & Após & $\begin{array}{l}\% \\
\text { dif }\end{array}$ & Antes & Após & $\begin{array}{l}\% \\
\mathrm{dif}\end{array}$ & Antes & Após & $\begin{array}{l}\% \\
\text { dif }\end{array}$ & Antes & Após & $\begin{array}{l}\% \\
\text { dif }\end{array}$ \\
\hline \multirow{8}{*}{ है } & Repouso & $77 \pm 3,3$ & $74 \pm 2,7$ & -3 & $73 \pm 3,4$ & $63 \pm 2,0$ & $-14^{*}$ & $74 \pm 3,4$ & $66 \pm 3,6$ & -11 & $71 \pm 3,1$ & $60 \pm 2,1$ & $-15 \#$ \\
\hline & pré-esforço & $81 \pm 3,0$ & $80 \pm 2,4$ & -1 & $83 \pm 3,3$ & $70 \pm 2,8$ & $-17^{*}$ & $77 \pm 3,3$ & $73 \pm 3,9$ & -5 & $79 \pm 3,1$ & $67 \pm 2,4$ & $-16 \#$ \\
\hline & 1 & $113 \pm 4,9$ & $113 \pm 4,8$ & 0 & $107 \pm 3,2$ & $101 \pm 2,8$ & -6 & $111 \pm 3,2$ & $103 \pm 4,2$ & $-7 \#$ & $115 \pm 3,4$ & $105 \pm 3,0$ & $-9 \#$ \\
\hline & 2 & $135 \pm 4,8$ & $135 \pm 4,2$ & 0 & $126 \pm 3,3$ & $118 \pm 2,5$ & $-6^{*}$ & $130 \pm 2,9$ & $122 \pm 4,0$ & $-6 \#$ & $131 \pm 3,5$ & $122 \pm 3,4$ & $-7 \#$ \\
\hline & 3 & $162 \pm 4,9$ & $163 \pm 4,6$ & 1 & $153 \pm 3,3$ & $151 \pm 2,7$ & -1 & $158 \pm 3,2$ & $155 \pm 3,3$ & -2 & $155 \pm 3,7$ & $151 \pm 3,2$ & -3 \\
\hline & 4 & $189 \pm 2,5$ & $190 \pm 2,2$ & 1 & $184 \pm 2,6$ & $184 \pm 2,6$ & 0 & $188 \pm 2,9$ & $184 \pm 2,7$ & -2 & $182 \pm 2,0$ & $182 \pm 2,0$ & 0 \\
\hline & máxima & $192 \pm 2,6$ & $192 \pm 2,0$ & 0 & $188 \pm 2,6$ & $191 \pm 2,4$ & 2 & $193 \pm 3,4$ & $194 \pm 3,4$ & 1 & $195 \pm 3,3$ & $196 \pm 1,7$ & 1 \\
\hline & rec & $126 \pm 4,2$ & $125 \pm 2,7$ & -1 & $124 \pm 3,4$ & $126 \pm 3,6$ & 2 & $121 \pm 5,1$ & $122 \pm 5,3$ & 1 & $126 \pm 3,1$ & $129 \pm 3,0$ & 2 \\
\hline \multirow{6}{*}{ 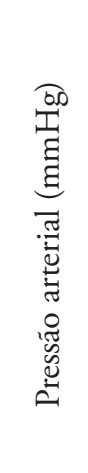 } & PAS sentado & $116 \pm 2,1$ & $118 \pm 1,7$ & 2 & $118 \pm 2,6$ & $119 \pm 2,4$ & 1 & $120 \pm 2,5$ & $122 \pm 2,7$ & 2 & $124 \pm 3,2$ & $123 \pm 2,3$ & 1 \\
\hline & PAD sentado & $75 \pm 1,9$ & $75 \pm 1,6$ & 0 & $74 \pm 1,8$ & $74 \pm 1,6$ & 0 & $77 \pm 1,3$ & $72 \pm 1,5$ & $-7 \#$ & $76 \pm 1,5$ & $71 \pm 1,7$ & $-7 \#$ \\
\hline & PASpré-esforço & $122 \pm 2,7$ & $123 \pm 2,3$ & 1 & $123 \pm 2,4$ & $120 \pm 2,4$ & -2 & $124 \pm 2,6$ & $123 \pm 2,5$ & -1 & $128 \pm 3,1$ & $122 \pm 2,2$ & $-5 \#$ \\
\hline & PADpré-esforço & $79 \pm 2,8$ & $75 \pm 1,5$ & -5 & $78 \pm 1,9$ & $74 \pm 1,5$ & $-5 \#$ & $79 \pm 1,3$ & $74 \pm 1,9$ & $-6 \#$ & $77 \pm 1,3$ & $71 \pm 1,8$ & $-8 \#$ \\
\hline & PASfim & $185 \pm 4,6$ & $186 \pm 3,9$ & 1 & $176 \pm 4,3$ & $192 \pm 2,8$ & $9^{*}$ & $191 \pm 3,5$ & $200 \pm 4,0$ & 5 & $178 \pm 6,4$ & $201 \pm 4,3$ & $13^{*}$ \\
\hline & PADfim & $71 \pm 1,8$ & $70 \pm 2,0$ & -1 & $79 \pm 2,5$ & $60 \pm 0,8$ & $-24^{*}$ & $78 \pm 2,6$ & $64 \pm 1,8$ & $-18^{*}$ & $78 \pm 2,3$ & $63 \pm 1,2$ & $-20^{*}$ \\
\hline \multirow{3}{*}{ 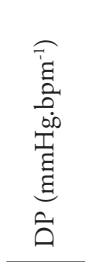 } & sentado & $8.9 \pm 4,2$ & $8.7 \pm 3,1$ & -2 & $8,7 \pm 4,5$ & $7,5 \pm 2,9$ & $-14 \#$ & $8,9 \pm 4,6$ & $8,0 \pm 4,5$ & -10 & $8,8 \pm 5,3$ & $7,4 \pm 2,8$ & $-16^{*}$ \\
\hline & pré-esforço & $9.9 \pm 4,2$ & $9.8 \pm 3,7$ & -1 & $10,3 \pm 5,5$ & $8,3 \pm 4,1$ & $-19^{*}$ & $9,5 \pm 4,0$ & $9,0 \pm 4,7$ & -6 & $10,1 \pm 5,3$ & $8,1 \pm 3,2$ & $-29^{*}$ \\
\hline & fim & $35,6 \pm 1,0$ & $35,6 \pm 7,8$ & 0 & $33,1 \pm 8,8$ & $36.6 \pm 7,0$ & $11^{*}$ & $36,9 \pm 1.0$ & $38,7 \pm 1.1$ & 5 & $34,9 \pm 1.7$ & $39,3 \pm 8,6$ & $13^{*}$ \\
\hline
\end{tabular}


Gcon = grupo controle; Gond = grupo ondulatório; Gcres = grupo crescente; Ges = grupo escalonado . ${ }^{*} p<0,05$ para Gcres vs Gesc;

${ }^{* *} p<0,01$ para Gcon vs Gond, Ges e Gcres - ANOVA de uma via.
Gcon = grupo controle; Gond=grupo ondulatório; Gcres = grupo crescente; Ges = grupo escalonado; * $p<0,05$ para Gcon vs Gond e Ges:

${ }^{* *} p<0,01$ para Gcres vs Gond;

\#p < 0,05 para Gcon vs Gond, Gcres e Ges. ANOVA de uma via.

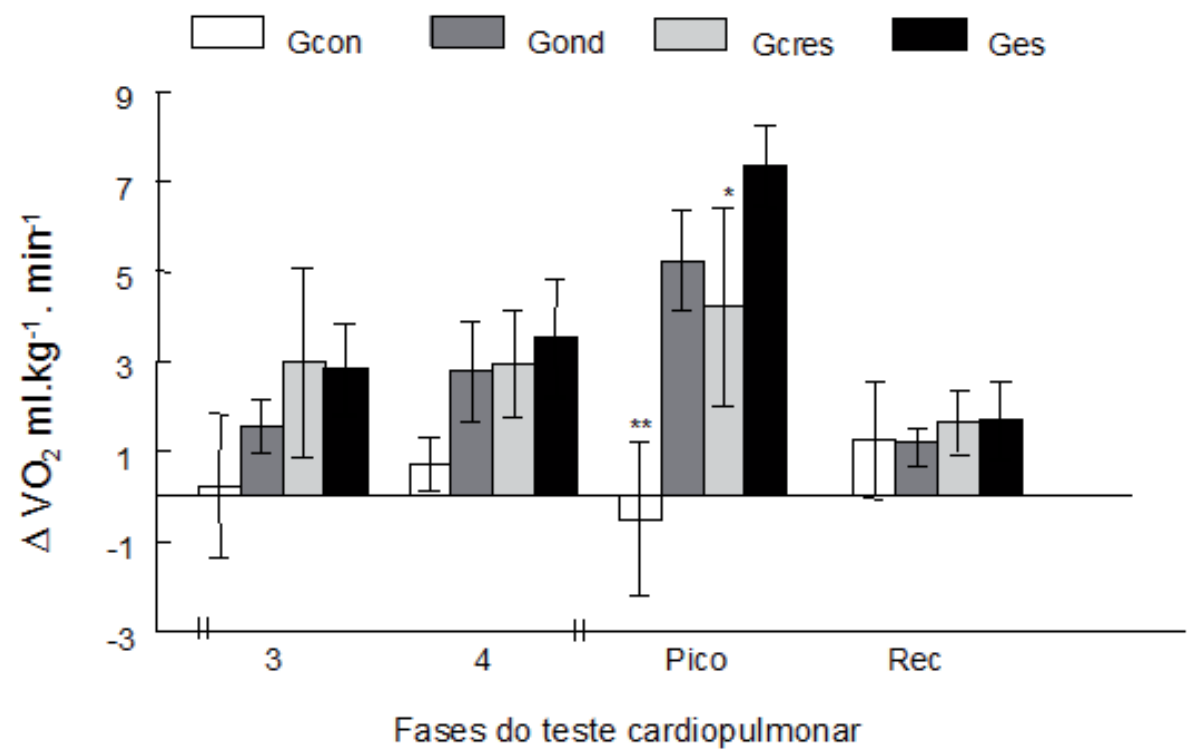

FIGURA 2 - Diferenças no consumo de oxigênio relativo $\left(\mathrm{ml}^{\mathrm{kg}} \mathrm{kg}^{-1} \cdot \mathrm{min}^{-1}\right)$ alcançados no teste de esforço antes e após o PTA periodizado com diferentes cargas em grupos de bombeirso militares/ES.

As variáveis cardiovasculares de $\mathrm{FC}$, PA e DP estão apresentados na TABELA 3. A FC reduziu-se significativamente $(\mathrm{p}<0,01)$ nos grupos Gond $\mathrm{e}$ Ges. Os resultados da FC do Gcres também foram reduzidos em $11 \%$ e $5 \%$, porém, essas diferenças não foram significativas $(\mathrm{p}>0,05)$.

A FC durante os estágios dos testes cardiopulmonares antes e após o PTA reduziu significativamente nos estágios 1 e 2 para os grupos Gcres e Ges, enquanto que para o Gond a redução ocorreu somente no estágio 2; desse estágio em diante nenhuma diferença foi demonstrada.
Nas comparações das diferenças entre as médias $(\Delta)$ antes e após o PTA, observa-se a magnitude dos aumentos ou reduçōes encontradas em cada variável. Sendo assim, os deltas da FC (FIGURA 3) foram negativos para os quatro grupos, com diferença significativa entre o Gcon "versus" os outros três grupos que treinaram, tanto na situação da medida no repouso, como em pré-esforço. Além disso, para pré-esforço, houve uma redução da FC apresentada pelo Gond, diferente em magnitude daquela apresentada pelo Gcres (14 bpm contra apenas $4 \mathrm{bpm}$, respectivamente). A redução do Ges foi de $12 \mathrm{bpm}$.

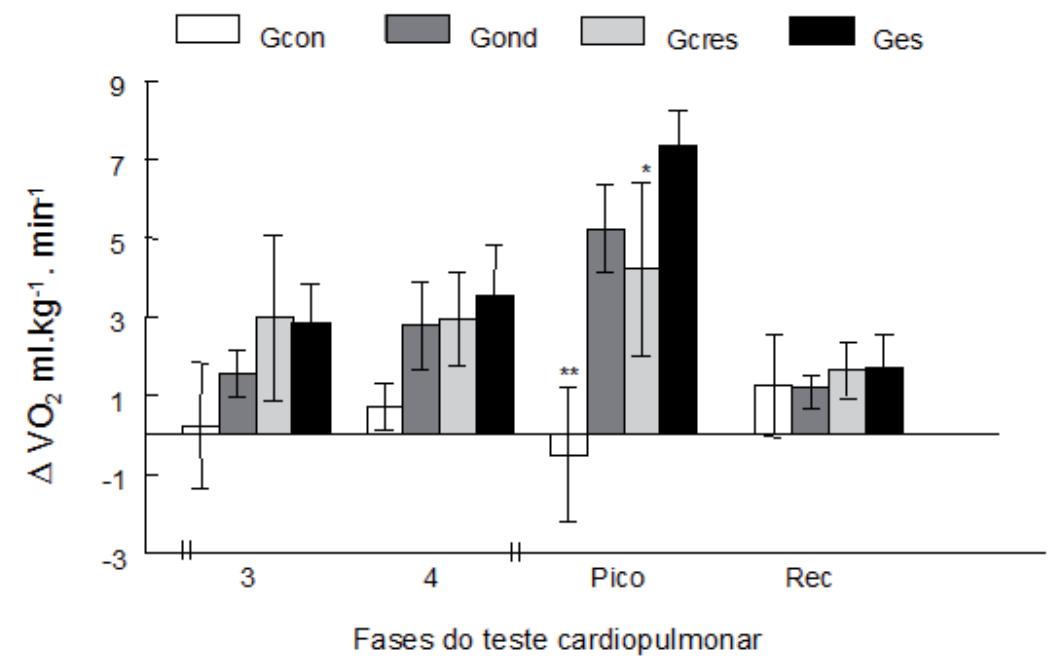

FIGURA 3 - Diferenças $(\Delta)$ da frequência cardiaca nas fases do teste cardiopulmonar antes e após o PTA de 13 semanas com diferentes periodizações de cargas em grupos de bombeiros militares/ES. 
Quanto à pressão arterial, a PAS no pré-esforço estava significativamente reduzida no teste após o PTA apenas para Ges. A PAD no repouso sofreu redução significativa $(\mathrm{p}<0,01)$ de $7 \%$ para ambos Gcres e Ges, e no pré-esforço em 6\% para o Gcres, e $8 \%$ no Ges (TABELA 3). Para Gond houve uma redução (5\%), porém não significativa. Quando comparados os deltas entre os grupos não foram encontradas diferenças significativas.

As medidas de PASfim e PADfim para os três grupos treinados tiveram aumentos de $9 \%, 5 \%$ e $13 \%$, respectivamente para Gond, Gcres e Ges, e reduçōes na PAD de 24\%, 18\% e 20\%. Exceto a PAS do Gcres, todas as diferenças foram estatisticamente significantes (TABELA 3). Os deltas ( $\Delta$ ) da PASfim, tanto do Gond como do Ges, foram significativamente maiores em relação ao Gcon. Já para a PADfim foram semelhantes entre os três grupos treinados.

Os valores calculados do DP (FIGURA 4) reduziram-se para os três grupos, sendo encontradas diferenças apenas para os grupos Gond e Ges. Porém, os $\Delta$ de redução foram semelhantes para os quatro grupos no repouso. No pré-esforço, foram significativamente diferentes entre Gcon vs Gond e Ges e entre Gcres vs Gond e Ges. O DPfim mostrou um aumento significativo para Gond, Gcres e Ges. Quanto aos $\Delta$ de DPfim, os aumentos mostrados para Gond e Ges foram significativamente maiores do que Gcon.

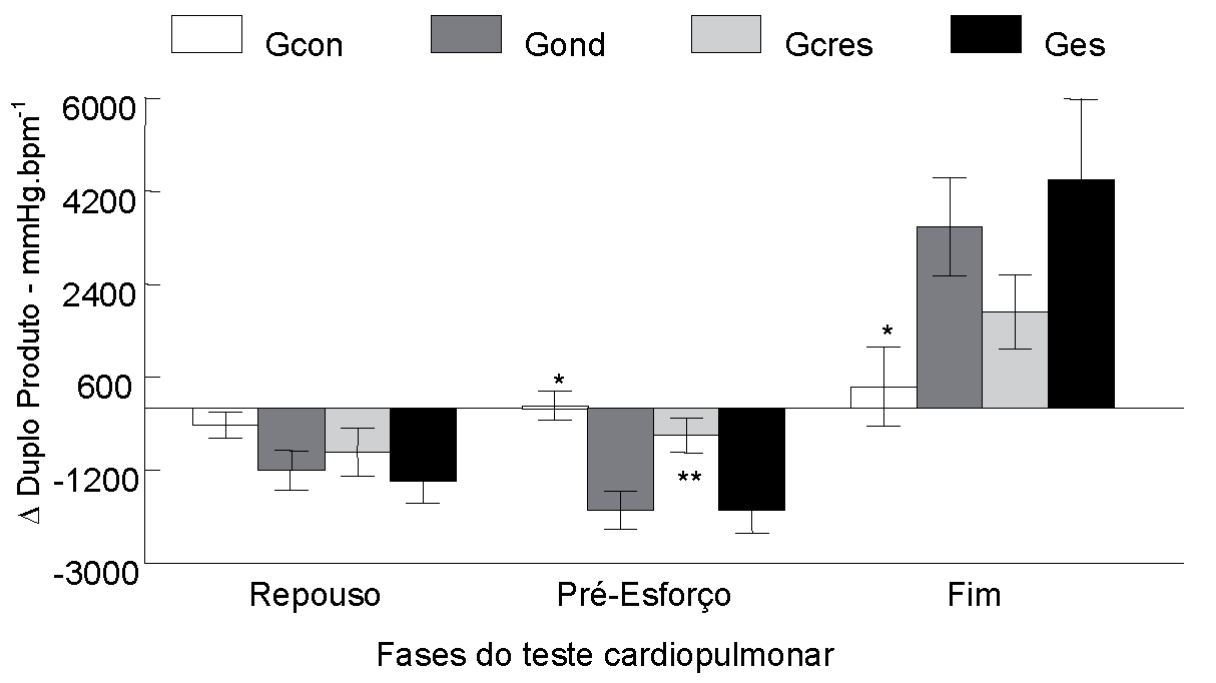

FIGURA 4 - Diferenças dos resultados do Duplo Produto (produto da PAS pela FC) atingidos nos testes cardiopulmonares antes e após o PTA com diferentes periodizações de cargas aplicados a bombeiros militares/ES.

\section{Discussão}

O principal objetivo deste trabalho foi estudar a influência da periodização com intensidades de cargas de um treinamento aeróbio sobre os efeitos proporcionados nas variáveis de composição corporal (\%G e $\Sigma 6 \mathrm{DC}$ ), $\mathrm{VO}_{2}$ em níveis de esforço submáximos e máximos, $\mathrm{FC}$ e PA, nas situaçōes de repouso, esforço e recuperação, e do duplo produto em: repouso, pré-esforço e ao final do esforço, coletados em teste cardiopulmonar.

Em relação à aplicação do PTA demonstrou-se a efetividade do treinamento por meio da análise das respostas adaptativas de FC e velocidades de corrida desenvolvidas nas sessões.

Considerando o poder do $\mathrm{VO}_{2}$ como medidor de sucesso deste tipo de treinamento ${ }^{7,22-23}$ e de sua relação com a intensidade ${ }^{9,24-26}$, os resultados do Gcres, mesmo tendo treinado mais tempo com as maiores intensidades do PTA, e consequentemente com exigências de consumo de oxigênio também maiores $^{27}$, não foram melhores do que dos outros dois grupos como se poderia esperar. Portanto, para os objetivos de melhoria no $\mathrm{VO}_{2}$ não foi necessário mais de três semanas com altas intensidades de esforços ( $85 \%$ a $90 \%$ da FCmáx.).

Neste trabalho, pôde-se identificar um estado de fadiga mais acentuado no Gcres, representado pela queda de rendimento (relação da velocidade de corrida com a FC nas sessões de treino) entre as semanas 10 e 11 (FIGURA 1). Este fenômeno pode ter ocorrido
Gcon = grupo controle; Gond = grupo ondulatório; Gcres = grupo crescente; Ges = grupo escalonado; ${ }^{*} p<0,05$ para Gcon vs Gond e Ges; ${ }^{* *} p<0,01$ para Gcres vs Gond e Ges. 
pelo acúmulo de cargas mais intensas, provavelmente acima do limiar ventilatório individual ${ }^{28}$. Além disso, mostra que a velocidade pode ser bem utilizada como avaliação do treinamento ${ }^{7}$.

Outros trabalhos também estudaram programas de treinamento tanto com cargas fixas como com cargas crescentes, mas não apresentaram discussões sobre a periodização das cargas ${ }^{29-31}$. É interessante notar que o $\mathrm{ACSM}^{5}$ não discute e nem apresenta uma proposta de periodização de cargas aeróbias, chamando atenção apenas para a necessidade de uma progressão gradual. Porém, apenas preocupação com a progressão gradual não garante que ocorram fases de estímulo e recuperação nas semanas, e muito menos uma eficácia maior para cargas mais intensas, como mostrado nesse estudo.

Em relação à composição corporal observa-se que o IMC não é um bom indicador para interpretação do efeito do treinamento aeróbio sobre a composição corporal. Nos três grupos treinados houve modificações significativas no $\% \mathrm{G}$ e no $\Sigma 6 \mathrm{DC}$, mas não no IMC. Os dados antropométricos aproximaram-se daqueles encontrados por outros autores para grupos de homens bombeiros ${ }^{32-33}$. Milesis et al. ${ }^{32}$, por exemplo, mostraram que a redução do $\% \mathrm{G}$ é dependente do tempo de duração dos estímulos em cada sessão, sendo 30 minutos o suficiente para esse objetivo. Para um aumento de $16 \%$ no $\mathrm{VO}_{2}$ máx., eles observaram uma redução de 5\% no \%G, após 20 semanas de treinamento com intensidades de $85-90 \%$ da FCmáx., o que foi confirmado neste trabalho.

Nenhuma referência foi encontrada em função de diferentes periodizações do treinamento aeróbio sobre a composição corporal. Os nossos dados mostram que realizar uma atividade aeróbia intensa durante um maior número de microciclos, como foi o caso do Gcres, não possibilita uma redução maior de gordura subcutânea. Por outro lado, uma relação entre a intensidade e o número de estímulos com cargas aeróbias nos microciclos parece ser importante, já que apenas Ges mostrou diferenças significativas em relação ao Gcon, quando comparadas os $\Delta$ das avaliaçôes antes e após o PTA.

Em relação aos principais achados cardiovasculares, os efeitos em repouso e pré-esforço apontam para uma PAD reduzida significativamente para os grupos Gcres (posição sentada) e para Gcres e Ges (pré-esforço), estando de acordo com a literatura $2,8,34$. WHELTON et al. ${ }^{34}$ concluíram, a partir de uma meta-análise que incluiu 54 ensaios clínicos, que o exercício aeróbio tem efeito de redução da PA de repouso, sendo $3,8 \mathrm{mmHg}$ para PAS e $2,5 \mathrm{mmHg}$ para a PAD. As reduçōes encontradas nos bombeiros militares deste estudo ficaram entre 5 e $8 \%$ ( 4 a $6 \mathrm{mmHg}$ ), sem diferença entre os grupos com relação à magnitude dos deltas.

Considerando que as respostas pressóricas não estavam alteradas no primeiro teste, as reduções em repouso e pré-esforço além dos $2 \mathrm{mmHg}$ de $\mathrm{PAD}$ poderiam diminuir o risco para doenças associadas com pressão sanguínea elevada ${ }^{34}$, o que seria um resultado importante para a condição de saúde dos praticantes desses tipos de estratégias de periodizaçóes e, mesmo para normotensos, poderia ser uma condição preventiva ${ }^{35}$. Como mecanismos prováveis estão queda da resistência periférica e um aumento do débito cardíaco, podendo ter ocorrido devido a atividade simpática reduzida ${ }^{36}$.

É interessante ainda observar a expressiva redução de PADfim para os três grupos, apontando para magnitudes interessantes em função do efeito do treinamento aeróbio sobre a PAD e com quedas significativas da pressão arterial média (PAM) de 111 para $104 \mathrm{mmHg}, 116$ para $109 \mathrm{mmHg}$, e de 111 para $109 \mathrm{mmHg}$, nos grupos Gond, Gcres e Ges, respectivamente. $\mathrm{O}$ Gcon teve a PAM semelhante em $109 \mathrm{mmHg}$. Apesar de melhorias da função do miocárdio, representada pela redução do DP e bradicardia nos três grupos treinados, o impacto dessas modificações não foram maiores no Gcres.

Os efeitos medidos pelo teste cardiopulmonar não foram diferentes entre os grupos treinados com relação à redução da $\mathrm{FC}$ nas fases submáximas até o estágio 3 , no aumento no DPfim, e nem no $\mathrm{VO}_{2}$ pico, mostrando novamente que Gcres não obteve vantagem por ter treinado mais semanas com as intensidades mais altas.

As evidências mostram que a redução da FC, induzida pelo treinamento, torna-se atenuada de acordo com o passar do tempo do programa ${ }^{37-38}$. Os resultados existentes na literatura sugerem que a bradicardia de treinamento é resultado de uma combinação de uma diminuição adaptativa da FC intrínseca e de uma influência autonômica, alterada na forma do número/responsividade de receptores adrenérgicos e um tono parassimpático aumentado ${ }^{39-42}$, e esse efeito persiste, tanto em repouso quanto em níveis submáximos, quando em condiçôes de bloqueio simpático e parassimpático ${ }^{43}$. Embora a bradicardia seja causada por aumento da atividade parassimpática ${ }^{3,44}$, durante o exercício, a redução da FC é proporcionada por uma diminuição dos estímulos simpáticos ${ }^{43}$, a não ser para atletas próximos a $100 \%$ do pico de desempenho ${ }^{45}$.

Isto exposto cabe inferir que o Gcres passava por um menor efeito de redução simpática no teste após o PTA, visto as elevadas respostas de FC e concomitante redução da velocidade de corrida encontrada nas 
semanas 11 e 12 . Além disso, ressalta-se que durante exercícios de alta intensidade em homens saudáveis foi mostrado que as influências vagais são retiradas e o "drive" adrenérgico torna-se o principal determinante da FC e de sua aceleração ${ }^{46}$. De acordo com NóbregA e ARAÚJO ${ }^{47}$, a magnitude dessa aceleração é aumentada em proporção com a intensidade e duração do esforço. Todos os três grupos realizaram esforços próximos a $80 / 85 \%$ do $\mathrm{VO}_{2}$ máx. nas últimas três semanas de treinamento, porém o Gcres treinou com essa intensidade durante nove semanas, o que poderia influenciar nas adaptações desse fenômeno de aceleração.

Dentro dessa lógica, algumas evidências indicam que atividades vigorosas são mais cardioprotetoras do que atividades moderadas, apesar de essa evidência ainda não ser totalmente convincente ${ }^{6}$. Uma atividade vigorosa para estímulos aeróbios pode estar dentro dos percentuais de $80-85 \%$ do $\mathrm{VO}_{2}$ máx., em respeito aos princípios da sobrecarga e adaptação, que sugerem que incrementos devem ser pequenos e um tempo adequado deve ser permitido para a adaptação.

$\mathrm{O} \mathrm{VO}_{2}$ submáximo encontrado nos estágios do teste para os três grupos não se alterou, ou se elevou ligeiramente, o que está de acordo com ATCHEN e JEUKENDRUP ${ }^{44}$, que relatam o efeito do treinamento aeróbio sobre redução do "slope" entre a relação $\mathrm{FC}-\mathrm{VO}_{2}$.

Considerando que o DP é uma medida prática clássica por não ser invasiva e é preditora do consumo de oxigênio do miocárdio ${ }^{48}$, os resultados de redução em repouso e ainda um maior DPfim em função da melhoria da aptidão física estão de acordo com os encontrados na literatura ${ }^{49}$.

Segundo HiCKSON et al. ${ }^{50}$ a não ser que o estímulo de treinamento seja aumentado, um programa diário de treinamento de alta intensidade não resulta em um efeito adicional ao aumento no $\mathrm{VO}_{2}$ máx. ou diminuição no lactato sanguíneo ou na FC, em resposta a um exercício submáximo após três semanas, tornando-se um programa de "manutenção". Igualmente, sugeriram um tempo de adaptação para os sistemas que limitam o $\mathrm{VO}_{2}$ máx. menor do que aproximadamente 10 dias.

Segundo Fry et al. ${ }^{10}$, a supercompensação das cargas pode não ocorrer, caso a capacidade de reposição de glicogênio muscular não seja efetiva. Isso pode ter ocorrido com o Gcres nas últimas três semanas de treinamento, quando a velocidade média das sessōes decresceu. Diferente dos grupos Gond e Ges, o grupo Gcres sofreu um acúmulo de cargas em função das semanas seguidas com intensidades mais fortes, de 85-90\% da FCmáx.

Com essas consideraçôes e os resultados encontrados neste trabalho, sugere-se um programa misto (ondulatório/escalonado), com cargas escalonadas de duas em duas semanas até se atingir cargas em torno de $85-90 \%$ da FCmáx., seguido de um ou dois microciclos recuperativos, iniciando-se um novo mesociclo.

Como fator de limitação desse estudo, sabemos que, segundo JONES e CARTER ${ }^{51}$, os efeitos do treinamento aeróbio podem ser caracterizados por quatro parâmetros da aptidão aeróbica: o $\mathrm{VO}_{2}$ máx., a economia de exercício/corrida, os limiares de lactato e ventilatório, e a potência crítica. Neste trabalho somente analisados o primeiro parâmetro o que pode limitar a nossa interpretação.

Ainda assim, este estudo se diferencia contribuindo com informações sobre a aplicação científica da periodização, o que pode beneficiar a maioria da população.

Finalmente, os resultados sugerem que, em relação aos três métodos de distribuição de intensidade de cargas, o crescente, com aumento em sequência de intensidade em $5 \%$ por semana e manutenção das cargas pelo restante do programa, não mostrou ser mais eficiente do que os outros dois métodos. Portanto, treinar com altas intensidades aeróbias (estímulos fortes) por mais tempo parece não oferecer vantagens nas adaptações fisiológicas em homens saudáveis. Dessa forma, preconiza-se a corrida com uma distribuição de cargas ao longo do tempo de forma escalonada e/ou ondulatória.

\begin{abstract}
Effects of different periodization models of aerobic training on cardiovascular parameters, metabolic and body composition in military firefighters

The aim of the present study was to analyze the effect of different periodization models of aerobic training on cardiovascular and metabolic parameters, and body composition. Sixty-five military firefighters y / ES (25.9 \pm 0.6 years) were divide into four groups: control (GCON, $n=15)$ without treatment; undulating $(G u, n=18)$, scaled $(G s, n=17)$ and increasing $(G i, n=15)$; they trained during 13 weeks, three weekly sessions of 30 minutes at intensities of $60 \%$ to $90 \%$ HRmax. Heart rate (HR) was monitored
\end{abstract}


in all sessions . Anthropometric measurements and cardiopulmonary exercise testing (Aerosport TEEM100) were conducted before and after the experimental program. A reduction in body fat percentage, bradycardia at rest and submaximal HR with increased oxygen consumption, and reduction of RPP, in $\mathrm{DBP}$ at rest for all three groups trained without advantages for $\mathrm{Gi}$, were observed. These results suggest that training programs which includes increasing and/or undulating aerobic training models may be more suitable for non-athletes.

KeY woRds: Physical preparation; Periodization; Heart rate; Cardiovascular health; Bradycardia; Physiological adaptation.

\section{Referências}

1. Haskell WL, Lee I-M, Pate RR, et al. Physical activity and public health: updated recommendations from the American College of Sports Medicine and the American Heart Association. Med Sci Sports Exerc. 2007;39: 1423-34.

2. Murray A, Delaney T, Bell C. Rapid onset and offset of circulatory adaptations to exercise training in men. J Hum Hypertens. 2006;20:193-200.

3. Zanesco A, Antunes E. Effects of exercise training on the cardiovascular system: pharmacological approaches. Pharmacol Ther. 2007;114:307-17.

4. O’Donovan G, Blazevich AJ, Boreham C, et al. The ABC of physical activity for health: a consensus statement from the British Association of Sport and Exercise Sciences. J Sports Sci. 2010;28:573-91.

5. Garber CE, Blissmer B, Deschenes MR, et al. Quantity and quality of exercise for developing and maintaining cardiorespiratory, musculoskeletal, and neuromotor fitness in apparently healthy adults: guidance for prescribing exercise. Med Sci Sports Exerc. 2011;43:1334-59.

6. Powell KE, Paluch AE, Blair SN. Physical activity for health: what kind? How much? How Intense? On top of what? Annu Rev Public Health. 2011;32:349-65.

7. Borresen J, Lambert MI. The quantification of training load, the training response and the effect on performance. Sports Med. 2009;39:779-95.

8. Kesaniemi YA, Danforth Junior E, Jensen MD, Kopelman PG, Lefebvre P, Reeder BA. Dose-response issues concerning physical activity and health: an evidence-based symposium. Med Sci Sports Exerc. 2001;33:S351-8.

9. Shephard RJ, Absolute versus relative intensity of physical activity in a dose-response context. Med Sci Sports Exerc. 2001;33:S400-18.

10. Fry RW, Morton AR, Keast D. Periodisation of training stress: a review. Can J Sport Sci. 1992;17:234-40.

11. Schiotz MK, Potteiger JA, Huntsinger PG, Denmark DC. The short-term effects of periodized and constant-intensity training on body composition, strength, and performance. J Strength Cond Res. 1998;12:173-8.

12. Issurin VB. Block periodisation versus traditional training theory: a review. J Sports Med Phys Fitness. 2008;48:65-75.

13. Issurin VB. New horizons for the methodology and physiology of training periodisation. Sports Med. 2010;40:189-206.

14. Fry RW, Morton AR, Keast D. Periodisation and the prevention of overtraining. Can J Sport Sci. 1992;17:241-8.

15. Oliveira AP. A influência da distribuição das cargas semanais de treinamento no rendimento aeróbio em não atletas [dissertação]. Rio de Janeiro (RJ): Universidade Gama Filho; 1995.

16. Karvonen J, Vuorimaa T. Heart rate and exercise intensity during sports activities: practical application. Sports Med. 1988;5:303-12.

17. Karvonen MJ, Kentala E, Mustala O. The effects of training on heart rate; a longitudinal study. Ann Med Exp Biol Fenn. 1957;35:307-15.

18. Novitsky S, Segal KR, Chatr-Aryamontri B, Guvakov D, Katch VL. Validity of a new portable indirect calorimeter: the AeroSport TEEM 100. Eur J Appl Physiol. 1995;70:104/1-6.

19. Wideman L, Stoudemire NM, Pass KA, McGinnes CL, Gaesser GA, Weltman A. Assessment of the Aerosport TEEM 100 Portable Metabolic Measurement System. Med Sci Sports Exerc. 1996;28:509-15.

20. Howley ET, Bassett Junior DR, Welch HG. Criteria for maximal oxygen uptake: review and commentary. Med Sci Sports Exerc. 1995;27:1292-1301. 
21. Day JR, Rossiter HB, Coats EM, Skasick A, Whipp BJ. The maximally attainable $\mathrm{VO}_{2}$ during exercise in humans: the peak vs. maximum issue. J Appl Physiol. 2003;95:1901-7.

22. Basset DR, Howley ET. Limiting factors for maximum oxygen uptake and determinants of endurance performance. Med Sci Sports Exerc. 2000;32:70-84.

23. Snell PG, Stray-Gundersen J, Levine BC, Hawkins MN. Maximal oxygen uptake as a parametric measure of cardiorespiratory capacity. Med Sci Sports Exerc.. 2007;39:103-7.

24. Leuenberger U, Sinoway L, Gubin S, Gaul L, Davis D, Zelis R. Effects of exercise intensity and duration on norepinephrine spillover and clearance in humans. J Appl Physiol. 1993;75:668-74.

25. Busso T. Variable dose-response relationship between exercise training and performance. Med Sci Sports Exerc. 2003;35:1188-95.

26. Swain D. Moderate or vigorous intensity exercise: which is better for improving aerobic fitness? Prev Cardiol. 2005;8:55-8.

27. Billat VL, Morton RH, Blondel NVL, et al. Oxygen kinetics and modelling of time to exhaustion whilst running at various velocities at maximal. Eur J Appl Physiol. 2000;82:178-87.

28. Wood RE, Hayter S, Rowbottom D, Stewart I. Applying a mathematical model to training adaptation in a distance runner. Eur J Appl Physiol. 2005;94:310-6.

29. Debusk RF, Stenestrand U, Sheehan M, Haskell WL. Training effects of long versus short bouts of exercise in healthy subjects. Am J Cardiol. 1990;65:1010-3.

30. Dart AM, Meredith IT, Jennings GL. Effects of 4 weeks resistance training on cardiac left ventricular structure and function. Clin Exp Pharmacol Physiol. 1992;19:777-83.

31. Goldsmith RL, Bigger JT, Steinman RC, Fleiss JL. Comparison of 24-hour parasympathetic activity in resistancetrained and untrained young men. J Am Coll Cardiol. 1992;20:552-8.

32. Milesis CA, Pollock ML, Bah MD, Ayres JJ, Ward A, Linnerud AC. Effects of different durations of physical training on cardiorespiratory function, body composition, and serum lipids. Res Q. 1976;47:716-25.

33. Davis PO, Dotson CO, Maria DLS. Relationship between simulated fire fighting tasks and physical performance measures. Med Sci Sports Exerc. 1982;14:65-71.

34. Whelton SP, Chin A, Xin X, He J. Effect of aerobic exercise on blood pressure a meta-analysis of randomized, controlled trials. Ann Intern Med. 2002;136:493-503.

35. Blair SN, Kohl Iii HW, Paffenbarger Junior RS, Clark DG, Cooper KH, Gibbons LW. Physical fitness and all-cause mortality: a prospective study of healthy men and women. JAMA. 1989:262:2395-2401.

36. Jennings G, Nelson L, Nestel P, et al. The effects of changes in physical activity on major cardiovascular risk factors, hemodynamics, sympathetic, function, and glucose utilization in man: a controlled study of four levels of activity. Circulation. 1986;73:30-40.

37. Swaine IL, Linden RJ, Mary DA. Loss of exercise training-induced bradycardia with continued improvement in fitness. J Sports Sci. 1994;12:477-81.

38. Kelley G, Tran ZV. Aerobic exercise and normotensive adults: a meta-analysis. Med Sci Sports Exerc. 1995;27:1371-7.

39. Negrão CE, Moreira ED, Brum PC, Denadai ML, Krieger EM. Vagal and sympathetic control of heart rate during exercise by sedentary and exercise-trained rats. Braz J Med Biol Res. 1992;25:1045-52.

40. Negrão CE, Moreira ED, Santos MCLM, Farah MAV, Krieger EM. Vagal function impairment after exercise training. J Appl Physiol. 1992;72:1749-53.

41. Catai AM, Chacon-Mikahil MPT, Martinelli FS, et al. Effects of aerobic exercise training on heart rate variability during wakefulness and sleep and cardiorespiratory responses of young and middle-aged healthy men. Braz J Med Biol Res. 2002;35:741-52.

42. Scott AS, Eberhard A, Ofir D, et al. Enhanced cardiac vagal efferent activity does not explain training-induced bradycardia. Auton Neurosci. 2004;112:60-8.

43. Moore RL, Korzik D. Cellular adaptations of the myocardium to chronic exercise. Prog Cardiovasc Dis. 1995;37:371-96.

44. Atchen J, Jeukendrup AE. Heart rate monitoring: applications and limitations. Sports Med. 2003;33:517-38.

45. Iellamo F, Legramante JM, Pigozzi F, et al. Conversion from vagal to ,ympathetic predominance with strenuous training in high-performance world class athletes. Circulation. 2002;105:2719-24.

46. Rowell LB. Human cardiovascular control. New York: Oxford University; 1993.

47. Nóbrega ACL, Araújo CGS. Heart rate transient at the onset of active and passive dynamic exercise. Med Sci Sports Exerc. 1993;25:37-41. 
48. Kitamura K, Jorgensen CR, Gobel FL, Taylor HL, Wang Y. Hemodynamic correlates of myocardial oxygen consumption during upright exercise. J Appl Physiol. 1972;32:516-22.

49. Hui SC, Jackson AS, Wier LT. Development of normative values for resting and exercise rate pressure product. Med Sci Sports Exerc. 2000;32:1520-7.

50. Hickson RC, Hagberg JM, Ehsani AA, Holloszy JO. Time course of the adaptive responses of aerobic power and heart rate to training. Med Sci Sports Exerc. 1981;13:17-20.

51. Jones AM, Carter H. The effect of endurance training on parameters of aerobic fitness. Sports Med. 2000;29:373-86.

ENDEREÇO

Anselmo José Perez

Centro de Educação Física e Desportos Universidade Federal do Espírito Santo

Av. Fernando Ferrari, 514 - Goiabeiras 29075-970 - Vitória - ES - BRASIL e-mail: anselmo@cefd.ufes.br
Recebido para publicação: 05/10/2012

1a. Revisão: 21/11/2012

2a. Revisão: 27/11/2012

3a. Revisão: 20/08/2013

Aceito: 28/08/2013 